\title{
Testing the evolution of the absolute magnitude of type Ia supernovae and cosmological parameters
}

\author{
Mahtessian A.P.*1, G.S. Karapetian , M.A. Hovhannisyan ${ }^{2}$, V.H. Movsisyan , and L.A. \\ Mahtessian $^{2}$ \\ ${ }^{1}$ NAS RA V. Ambartsumian Byurakan Astrophysical Observatory (BAO), Armenia \\ ${ }^{2}$ NAS RA Institute of Applied Problems of Physics, Armenia
}

\begin{abstract}
Computer simulations show that, in estimating cosmological parameters, the best agreement between theory and observation is achieved by assuming the evolution of the absolute magnitude of type Ia supernovae. This requires only $0.3 \mathrm{~m}$ of evolution for the time corresponding to $\mathrm{z}=1$. This leads to zero density of hidden energy in the Universe.
\end{abstract}

Keywords: cosmological models - supernovae - dark energy - dark matter: general

\section{Introduction}

One of the tasks in observant cosmology is to test cosmological hypotheses. Now, the most common hypothesis the Lambda CDM model, or the Friedman model. Cosmological models allow us to estimate the distance of distant objects in the universe. To test these models, you need distance standards. Such standards are considered type Ia supernovae.

To determine the distance using a simple formula:

$$
M=m-5 \lg D_{L}-25,
$$

where $m$ is the apparent magnitude, $M$ is the absolute magnitude, and $D_{L}$ is the luminosity distance. In the flat Friedman Universe, the luminosity distance of an object at a given $\mathrm{z}$ is estimated by the following formula (Carroll et al., 1992, Weinberg, 2008) :

$$
D_{L}=\frac{C(1+z)}{H_{0}} \int_{0}^{z} d z\left[(1+z)^{2}\left(1+\Omega_{M} z\right)-z(2+z) \Omega_{\Lambda}\right]^{-1 / 2}
$$

Where $\Omega_{\Lambda}+\Omega_{M}=1$. $\Omega_{\Lambda}$ is the fraction of dark or hidden energy and $\Omega_{M}$ is the mass fraction (hidden + visible mass).

We need one more formula for the model of the Universe with a zero cosmological constant $\left(\Omega_{\Lambda}=0\right)$. In this case, Mattig (1958) precisely solved the Einstein field equations and obtained the following relation:

$$
D_{L}=\frac{C}{H_{0} q_{0}^{2}}\left[q_{0} z+\left(q_{0}-1\right)\left(\sqrt{1+2 q_{0} z}-1\right)\right]
$$

Where $q_{0}$ is the deceleration parameter, in this case:

$$
q_{0}=\frac{\Omega_{M}}{2}
$$

In the case of a flat universe

$$
q_{0}=\frac{\Omega_{M}}{2}-\Omega_{\Lambda}
$$

When calculating the distance according to the formula $M=m-5 \lg D_{L}-25$ it is necessary to accurately assess the value of the apparent magnitude of the object (taking into account galactic absorption, Kcorrection, spectral region, etc.). The absolute magnitude must be known either from theoretical approaches or from empirical dependencies.

*amahtes@bao.sci.am, Corresponding author 


\section{Results}

\subsection{The case of the distance-independent absolute magnitude of supernovae SNe Ia}

In Figure 1 shows the Hubble diagram for the Union2 sample used by Amanullah et al. (2010). The sample consists of 557 stars, the observational data of which are carefully processed. We used exactly the same stars with the same observational data, i.e., we did not revise anything in the observational aspect. For the best approximation of the observational data, the authors obtained

$$
\Omega_{\Lambda}=0.73, \Omega_{M}=0.27
$$

Now let's see this result, how much is consistent with the assumption that type Ia supernovae are distance indicators. In Figure 2 shows the dependence of the absolute magnitude of these stars on the redshift for the obtained model parameters.

As you can see there is a clear dependence.

Now we find the value of these parameters at which the absolute magnitude of these stars does not depend on redshift.

In Figure 3 shows the dependence of the absolute magnitude of these stars on the redshift for the case $\Omega_{\Lambda}=0.42, \Omega_{M}=0.58$

Apparently there are no dependencies.

Now back to the Hubble diagram. It is clear from the diagram that the sum of the squared deviations of the observational data from the theoretical curve is clearly less for the case $\Omega_{\Lambda}=0.42, \Omega_{M}=0.58$ (Chi2 $=83.96)$, compared with the case $\Omega_{\Lambda}=0.73, \Omega_{M}=0.27($ Chi2 $=90.37)$. (This means that the authors did not conduct the most probable curve within the framework of the model under discussion.)

On the Hubble diagram, a model of the Universe with a zero cosmological constant is also discussed. Approximation gives $q_{0}=0.184$. Let's see what the diagram of the dependence of the absolute magnitude on the redshift for this model will give (Fig. 4).

It turns out that there is also no dependency between these quantities. Also, a smaller standard deviation is obtained than for the model with $\Omega_{\Lambda}=0.73, \Omega_{M}=0.27$.

Thus, we can say that the study of the dependence of the absolute magnitude of type Ia supernovae (also of any objects that are considered distance indicators) on redshift within the framework of the model under discussion is very important. This diagram and the Hubble c diagram complement each other and give consistent results. A comparison of two models (the Friedman's model of a flat universe and the model of the Universe with a zero cosmological constant) based on different subsamples of supernova stars from the Union (Kowalski et al., 2008) and Union2 (Amanullah et al., 2010) compilations using our absolute magnitude test was carried out in detail by Mahtessian et al. (2020).

Thus, assuming that the absolute magnitude of the supernova SNe Ia does not depend on distance (i.e. does not evolve), we get:

1. In the flat Universe of Friedman, the widespread opinion about the prevalence of hidden energy over mass is rejected. The maximum possible fraction of hidden energy is 0.5 (Mahtessian et al., 2020).

2. The model of the Universe with a zero cosmological constant at least describes the Universe no worse than the generally accepted Friedmann model of a flat Universe with a positive cosmological constant.

\subsection{The case of the distance-depending absolute magnitude of supernovae SNe Ia}

Let us assume that the absolute magnitude of SNe Ia supernovae is not constant, but changes with distance (there is an evolution of supernova luminosities). Let's see how the agreement between the observed and theoretical data improves? We will assume that the dependence on the distance of the absolute magnitude of supernovae is linear, i.e.

$$
M=M_{0}+\epsilon_{c} z
$$

Where $M_{0}=M(z=0)$.

We will study the SNe Ia Union2 sample (Amanullah et al. 2010). Computer simulation data are given in Tables 1 and 2. In the first case (Table 1), the case is considered without assuming the evolution of the absolute magnitude of supernovae. And in the second (Table 2) with the assumption of the evolution of the absolute magnitude of supernovae.

It can be seen from Tables 1 and 2 that under the assumption of the evolution of supernovae Ia, the observational data agree better with the theory (Chi2 is smaller) than under the assumption of a constant 
absolute magnitude. In this case, the density of the hidden energy turns out to be 0 , that is, the Universe consists only of gravitational matter. The same is obtained for the model of the Universe with zero cosmological constant (Tables 3 and 4 ).

In the framework of the $\Lambda \mathrm{CDM}$ model, in the case of a flat universe, the influence of the absolute magnitude $\mathrm{M}$ of type Ia supernovae on cosmological parameters has also been investigated. In particular, it was found that a change in this value by only $0.4^{m}$ leads to a change in the parameters from $\Omega_{\Lambda}=0.7$ and $\Omega_{M}=0.3$ to $\Omega_{\Lambda}=0$ and $\Omega_{M}=1$.

According to numerous studies, $M_{0}$ fluctuates in a very wide range from -18 to -19.5. It is clear that with such a strong dependence of $\Omega_{\Lambda}$ and $\Omega_{M}$ on M, this interval is very wide. This means that a correct estimate of the absolute magnitude $\mathrm{M}$ of type Ia supernovae is extremely important for estimating cosmological parameters.

As seen above, for the best agreement between the observational data and theory, the evolution of the absolute magnitudes of type Ia supernovae is required by only $0.3 \mathrm{~m}$ at a distance of $\mathrm{z}=1$. (Such a value of the evolution of the absolute magnitudes of supernovae is consistent with many observational results (for example, Kang et al., 2020), which is much less than the value of this interval. In this case, it turns out that the density of hidden energy in the universe is zero.

\section{Conclusion}

Thus, the universe is mainly composed of gravitational matter. Space is flat - Euclidean. The existence of hidden energy is rejected.

\section{References}

Amanullah R., et al., 2010, Astrophys. J. , 716, 712

Carroll S. M., Press W. H., Turner E. L., 1992, Ann. Rev. Astron. Astrophys. , 30, 499

Hubble E., 1929, Proceedings of the National Academy of Science, 15, 168

Kang J.-G., Gong Y., Cheng G., Chen X., 2020, Research in Astronomy and Astrophysics, 20, 055

Kowalski M., et al., 2008, Astrophys. J. , 686, 749

Mahtessian A. P., Karapetian G. S., Movsisyan V. H., Mahtessian L. A., 2020, Advances in Astrophysics, 5 , 18

Mattig W., 1958, Astronomische Nachrichten, 284, 109

Perlmutter S., et al., 1999, Astrophys. J. , 517, 565

Riess A. G., et al., 1998, Astron. J. , 116, 1009

Sandage A., Tammann G. A., 1982, Astrophys. J. , 256, 339

Weinberg S., 2008, Cosmology, Oxford University Press 
Table 1. The result of searching for the values of the parameters $M_{0}, \Omega_{\Lambda}, \Omega_{M}$ for a Flat Universe $\left(\Omega_{\Lambda}+\Omega_{M}=\right.$ 1) without assuming the evolution of the absolute magnitude of supernovae.

\begin{tabular}{llllll}
\hline Parameter & Variable & $\begin{array}{l}\text { Search } \\
\text { range }\end{array}$ & $\begin{array}{l}\text { Parameter } \\
\text { search } \\
\text { range }\end{array}$ & Chi $^{2}$ & $R^{2}$ \\
\hline$M_{0}$ & Yes & $-19.5 \div-18.0$ & -18.903 & & \\
$\epsilon_{c}$ & No & 0 & 0.000 & 83.74 & 0.9836 \\
$\Omega_{\Lambda}$ & Yes & $0 \div 1$ & 0.397 & & \\
$\Omega_{M}$ & Yes & $0 \div 1$ & 0.603 & & \\
\hline
\end{tabular}

Table 2. The result of searching for the values of the parameters $M_{0}, \Omega_{\Lambda}, \Omega_{M}$ for a Flat Universe $\left(\Omega_{\Lambda}+\Omega_{M}=\right.$ 1) with assuming the evolution of the absolute magnitude of supernovae.

\begin{tabular}{llllll}
\hline Parameter & Variable & $\begin{array}{l}\text { Search } \\
\text { range }\end{array}$ & $\begin{array}{l}\text { Parameter } \\
\text { search } \\
\text { range }\end{array}$ & $C h i^{2}$ & $R^{2}$ \\
\hline$M_{0}$ & Yes & $-19.5 \div-18.0$ & -18.875 & & \\
$\epsilon_{c}$ & Yes & $-1 \div 1$ & 0.304 & 83.23 & 0.9837 \\
$\Omega_{\Lambda}$ & Yes & $0 \div 1$ & 0.000 & & \\
$\Omega_{M}$ & Yes & $0 \div 1$ & 1.000 & & \\
\hline
\end{tabular}

Table 3. The result of searching for the values of the parameters $M_{0}, q_{0}$ for a universe with zero cosmological constant without the assumption of the evolution of the absolute magnitude of supernovae.

\begin{tabular}{llllll}
\hline Parameter & Variable & $\begin{array}{l}\text { Search } \\
\text { range }\end{array}$ & $\begin{array}{l}\text { Parameter } \\
\text { search } \\
\text { range }\end{array}$ & $C h i^{2}$ & $R^{2}$ \\
\hline$M_{0}$ & Yes & $-19.5 \div-18.0$ & -18.881 & & \\
$\epsilon_{c}$ & No & 0 & 0.000 & 83.28 & 0.9837 \\
$q_{0}$ & Yes & $0 \div 0.5$ & 0.184 & & \\
\hline
\end{tabular}

Table 4. The result of searching for the values of the parameters $M_{0}, q_{0}, \epsilon_{c}$ for a universe with zero cosmological constant with the assumption of the evolution of the absolute magnitude of supernovae.

\begin{tabular}{llllll}
\hline Parameter & Variable & $\begin{array}{l}\text { Search } \\
\text { range }\end{array}$ & $\begin{array}{l}\text { Parameter } \\
\text { search } \\
\text { range }\end{array}$ & $C h i^{2}$ & $R^{2}$ \\
\hline$M_{0}$ & Yes & $-19.5 \div-18.0$ & -18.875 & & \\
$\epsilon_{c}$ & Yes & $-1 \div 1$ & 0.304 & 83.23 & 0.9837 \\
$q_{0}$ & Yes & $0 \div 0.5$ & 0.500 & & \\
\hline
\end{tabular}




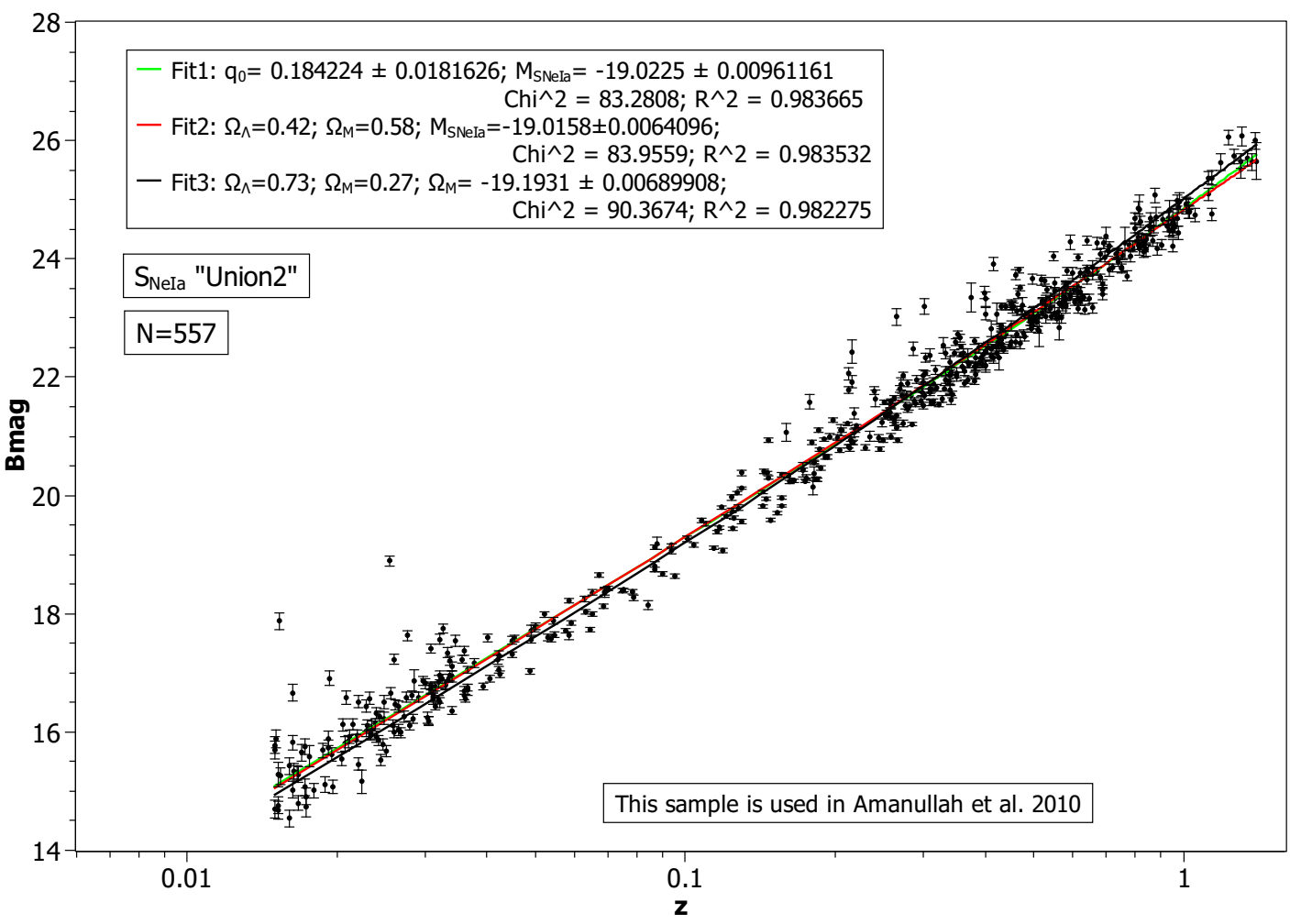

Figure 1. Hubble diagram for SNIa Amanullah et al. (2010) sample.

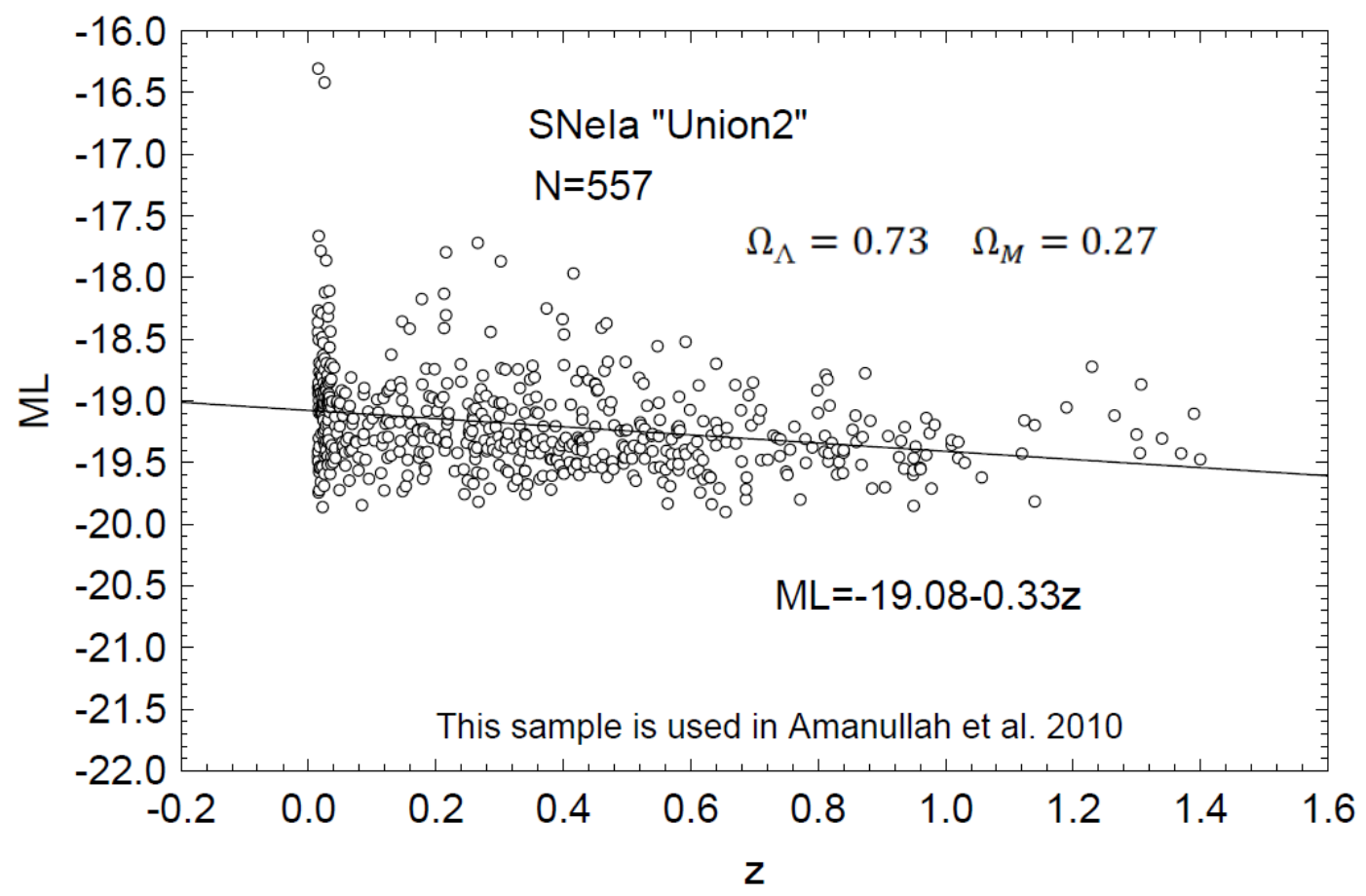

Figure 2. Absolute magnitude dependence on redshift forAmanullah et al. (2010) sample. 


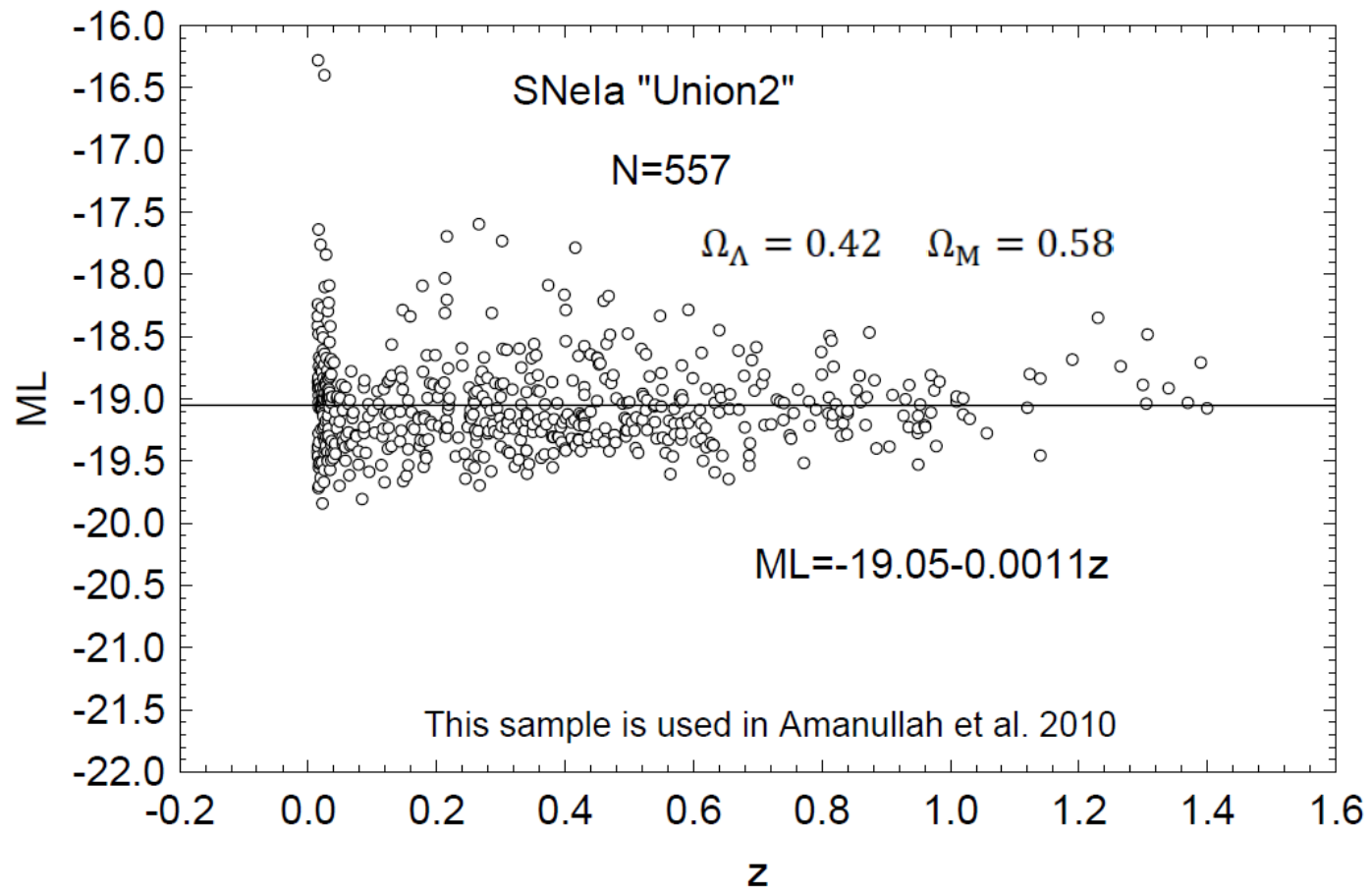

Figure 3. Absolute magnitude dependence on redshift for Amanullah et al. (2010) sample for the case $\Omega_{\Lambda}=0.42, \Omega_{M}=0.58$.

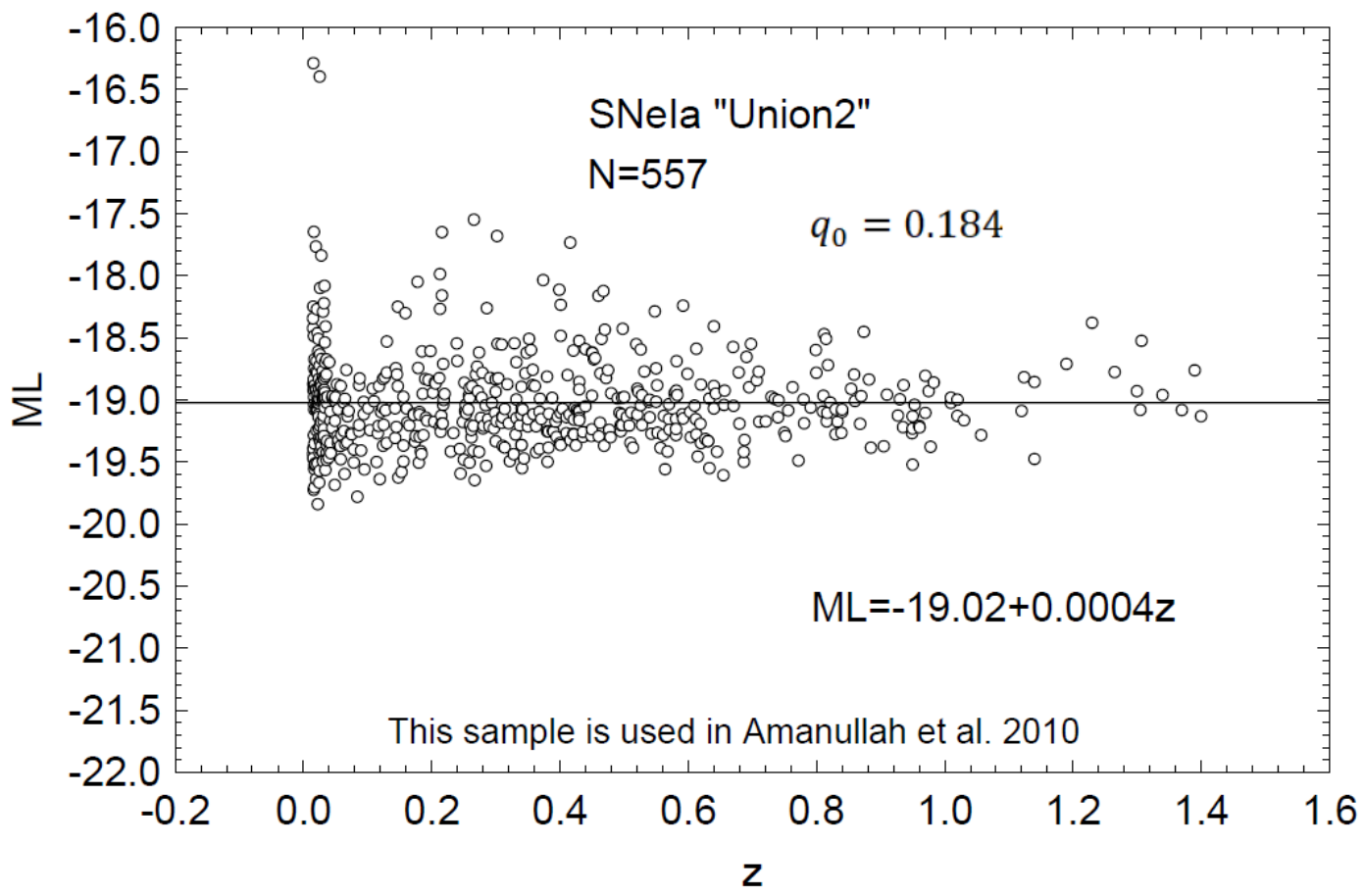

Figure 4. Absolute magnitude dependence on redshift for Amanullah et al. (2010) sample for a model of the Universe with a zero cosmological constant. 